\title{
The prosegment catalyzes native folding of Plasmodium falciparum plasmepsin II
}

\begin{abstract}
Plasmepsin II is a malarial pepsin-like aspartic protease produced as a zymogen containing an $\mathrm{N}$-terminal prosegment domain that is removed during activation. Despite structural similarities between active plasmepsin II and pepsin, their prosegments adopt different conformations in the respective zymogens. In contrast to pepsinogen, the proplasmepsin II prosegment is 80 residues longer, contains a transmembrane region and is non-essential for recombinant expression in an active form, thus calling into question the prosegment's precise function. The present study examines the role of the prosegment in the folding mechanism of plasmepsin II. Both a shorter (residues 77-124) and a longer (residues 65-124) prosegment catalyze plasmepsin II folding at rates more than four orders of magnitude faster compared to folding without prosegment. Native plasmepsin II is kinetically trapped and requires the prosegment both to catalyze folding and to shift the folding equilibrium towards the native conformation. Thus, despite low sequence identity and distinct zymogen conformations, the folding landscapes of plasmepsin II and pepsin, both with and without prosegment, are qualitatively identical. These results imply a conserved and unusual feature of the pepsin-like protease topology that necessitates prosegment-assisted folding.
\end{abstract}

Keyword: Plasmepsin II; Aspartic protease; Protein folding; Kinetic stability; Foldase 